\title{
A Proxy Culture
}

\author{
Luciano Floridi ${ }^{1}$
}

Published online: 21 October 2015

(C) Springer Science+Business Media Dordrecht 2015

We do not check out a hotel personally; we rely on TripAdvisor. We may have never met a person, yet we are 'friends' on Facebook. We may press 'like' but engage only in some kind of slacktivism. It does not matter whether we haven't got a clue about how to reach a place downtown, as long as we have access to Google Maps and follow the instructions. Five stars on Amazon may be sufficient to convince us of the quality of a product, even if we have never tried it ourselves. Being a 'best seller' in The New York Times Best Seller list is often a self-fulfilling prophecy.

In all these cases, something (the signifier) signifies something else (the signified). Such 'signifying' is at the heart of every semantic and semiotic process. It is the immensely important relation of 'standing for'. There is no sense, reference, or meaning without it. So, we have always helped ourselves to different kinds of signifying means, in order to interact with each other and the world, and make sense of both. We are the symbolic species after all, and twentieth-century philosophy-whether hermeneutically oriented or based on a philosophy of language - can easily be read in terms of a theory of signification. All this is clear, if complicated. The point here is that only our own culture, the culture that characterises mature information societies, is now evolving from being a culture of signs and signification into a culture of proxies and interaction. What is the difference? Why is this happening today? And what are the implications of such a major transformation? In order to answer these questions, one needs to understand better what a proxy is and what 'degenerate' proxies may be.

Let me start from the concept of proxy. In the Roman Catholic Church, a vicar is a representative or deputy of a bishop. This role, and its long familiarity, led to the idea of something being 'vicarious' as something 'acting or done for another' and, hence, 'vicariously'. The idea of 'proxy' is similar. The main difference is that its roots are political, not religious, for it is a late Middle English contraction of 'procuracy', which means 'legitimate action taken in the place of, or on behalf of, another', in the context of government or some kind of socio-political structures (e.g. one could get married by proxy). Today, in a vocabulary more deeply affected by information technology than by

Luciano Floridi

luciano.floridi@oii.ox.ac.uk

1 Oxford Internet Institute, University of Oxford, 1 St Giles, Oxford OX1 3JS, UK 
religious or political ideas, the term 'proxy' is likely to evoke thoughts of systems (e.g. a website) that accept requests for some service and passes them on to another system (e.g. the Internet). Yet, the meaning and the underlying experience are very similar. 'Vicariously' or 'by proxy', both qualify actions that are possible because something represents and replaces (acts or behaves instead of) something else.

Let us consider next the concept of a 'degenerate proxy'. Unfortunately, 'degenerate' has acquired a negative, evaluating meaning that in itself does not have to convey. In this context, I intend to use it in the purely mathematical sense according to which a degenerate case is a limiting case in which an object changes its nature so as to belong to another, usually simpler, class; for example, a point is a degenerate sphere of zero volume obtained when the radius of the sphere reaches zero. One can adapt this conceptual framework to make sense of 'degenerate proxies'. Recall that $\mathrm{P}$ is a proxy for $\mathrm{R}$ if and only if $\mathrm{P}$ both 'stands for' $\mathrm{R}$ and can 'stand in for' $\mathrm{R}$. Let us call this twofold relation the vicarious relation. If $\mathrm{P}$ has a vicarious relation with (i.e. it is a proxy for) $\mathrm{R}$ with a zero degree of 'standing in for' $\mathrm{R}$, then $\mathrm{P}$ is a degenerate proxy that only stands for, but cannot behave on behalf, or act instead, of R. Such degenerate proxies are signs. Similarly, if $\mathrm{P}$ has a vicarious relation with $\mathrm{R}$ but now with a zero degree of 'standing for' $\mathrm{R}$, then $\mathrm{P}$ is another kind of degenerate proxy, one that only behaves on behalf, or acts instead, of $\mathrm{R}$ without referring to it. Such degenerate proxies are surrogates. Let me elaborate.

According to a classic analysis by Charles Sanders Peirce, one may distinguish between three kinds of signs. Icons are signs that resemble what they stand for, e.g. a photograph of dark clouds is an icon. Indexes are signs that correlate with what they stand for, e.g. dark clouds are an index of impeding rain. Finally, symbols are signs that denote what they stand for by virtue of some convention, e.g. the English word 'cloud' is a symbol that denotes a specific meteorological phenomenon. In each case, the signifiers - the photograph, the dark clouds, the word 'cloud' - have a more or less complex relation of 'standing for' with respect to their referents, but they are unified by the fact that none of them can actually act on behalf of or instead of it. In other words, they are degenerate proxies, which have a zero-degree relation of 'standing in' with respect to their referents.

There seems to be a universal stage in any culture when degenerate proxies are mistaken for proxies, through a magical interpretation of signs. Icons, indexes, and symbols are endowed with the power of standing in for that which they only stand for and hence treated as if they were proxies. So that, for example, what one does to the photograph (icon) of a person is supposed to affect that person; bleeding that person, even in the rare case in which this is done because of hypertension (index) due to a chronic kidney disease, is supposed to cure the disease itself; and cursing the name of a person (symbol) is supposed to be a way of hurting that person. The theological debate on transubstantiation - the conversion of the bread and wine into the body and blood of Christ in the Eucharist - is a debate about whether the bread and wine are genuine proxies or mere degenerate proxies (i.e. signs) of Christ's bodily presence.

We saw that we have a different kind of degenerate proxy when the vicarious relation has a zero degree of standing for, transforming a proxy into a surrogate. Take chicory coffee. This is a classic coffee substitute that contains no caffeine but that is used to imitate and replace coffee. Chicory coffee usually does not have a semiotic relation of standing for real coffee. It is not a sign for coffee, in the same way 
as a picture (icon), smell (index), or name (symbol) of some coffee is. But it has a 'standing in for' relation with coffee because you can actually drink chicory coffee as a substitute for real coffee, in theory without even knowing that there is something else of which that chicory coffee is a surrogate.

To summarise, proxies are pragmatically more than signs because they are signifiers that also stand in for the signified and so you can interact with them instead of interacting with the signified. And they are epistemologically more than surrogates, because they are signifiers you can interact with that refer to the signified they replace, so you can still perceive the difference.

We have never been magic because signs are degenerate proxies, yet magic is exactly what we may become because of our proxy culture. This is a risk for those who mistake magic effects - the possibility of interacting with proxies that now do stand for and in for their referents - for magic causes or explanations (the supernatural, the paranormal, etc.). It also follows that semiotics, the discipline that studies signs, is really the study of only one kind of degenerate proxy, that is, a branch of proxiology, a yet to be developed discipline that studies the whole field of proxies, including degenerate proxies like signs and surrogates, as well as the use of proxies in social contexts (e.g. in signalling strategies).

And because signs are degenerate proxies, it makes sense to see our proxy culture as a direct development of the semiotic, symbolic, or sign-based culture fostered by the mass media in our recent past. The difference is that in a Newtonian world of analogue consumerism offline, in which purchase and ownership of physical goods is largely predominant, proxies were difficult to develop and less necessary in order to interact with the environment. But today, living onlife and in the infosphere, so completely immersed in such a bottomless sea of data, when renting and usage of services is at least as important as ownership of goods, proxies are both easy to obtain and necessary to use because they share a digital nature with their referents. It is elementary to have some informational structure standing for and in for some other informational structure, exactly in the same way as it is elementary to have the same digital 'stuff' work as software and as data for the software. Such an ontological uniformity has both facilitated the emergence of a proxy culture and made it vital as a solution to a problem that such culture itself poses, self-reinforcingly. It is estimated that humanity accumulated less than 1 zettabyte of data in the course of its entire history until 2009, but this figure has already reached 8 zettabytes this year and is expected to grow to 35 zettabytes by 2020. Admittedly, a lot of the data surrounding us may be utterly meaningless or worthless, but there is still a mind-boggling and constantly growing amount of valuable information available today, on any topic and any facet of any topic. This means that it is increasingly difficult to navigate in the infosphere without relying on proxies and, at the same time, that proxies are not just the solution but also part of the problem, given that they are a source of even more data, for which more proxies will be needed. The result is that the distance between us and the signified realities is quickly growing, and so is the need to shorten it through ever more signifiers with which we can interact effectively. Signs and 'signifying' are crucial phenomena in every culture, but only ours is a proxy culture because only now has quantity made a significant difference in quality, while technology has made possible genuine relations of standing in for something else as a matter of ordinary experience. 
There are inevitably some risks in all this. As I indicated above, we may misunderstand signs for proxies and fall into some kind of occultism. Or we may misunderstand surrogates for proxies and embrace some kid of 'shallowism'. In the same way as a culture of signs may be a self-referential culture, in which words only refer to words recursively, and never reach the world or their non-semantic referents, likewise, a proxy culture may become an ersatz culture, in which proxies become mere surrogates that not only hide their original references (the 'real' coffee) but make it hard or even impossible to reach it because they fully replace it without any residual link to an alternative reality. A world in which there is no chicory coffee is not a better world, but a world in which there is only chicory coffee is a worse, shallower world.

At the same time, a proxy culture can be an augmented culture, which offers more and better opportunities for our flourishing. For proxies in themselves have the potential to enable previously unmanageable or even impossible interactions and experiences (for example, both Etsy and Amazon have made it possible to sell and buy handmade goods online and globally, not just offline and locally). A good reminder is that, in statistics, a proxy is actually a variable that is irrelevant in itself but that serves in place of another variable that is otherwise unobservable or not measurable. If proxies can serve as bridges towards otherwise hard to access or even inaccessible spaces of experiences then our proxy culture will be a better, enhanced culture.

We are the amphibian generation that is moving out of the analogue world to live in a digital environment. No generation before has ever been forced to adapt so dramatically, to such profound changes, in such a short time. Part of our evolution will also concern how we design the proxies that will increasingly populate our infosphere and mediate our experiences; how we learn to interact healthily with them; and how we are going to control those who will be in charge of them. Whether our proxy culture will be an augmented one, instead of turning into an ersatz one, is one of the open challenges confronting any mature information society today. 